\title{
"SÓ MAIS UM MINUTINHO, TEACHER": PLANEJAMENTO ESTRATÉGICO COLABORATIVO E INDIVIDUAL PARA TAREFAS ORAIS EM L2 EM UMA ESCOLA PÚBLICA
}

\author{
Rafael Zaccaron ${ }^{1^{*}}$ \\ Donesca Cristina Puntel Xhafaj ${ }^{{ }^{\star *}}$ \\ Raquel Carolina de Souza Ferraz D'Ely ${ }^{1 * * *}$ \\ ${ }^{1}$ Universidade Federal de Santa Catarina, Florianópolis, SC, Brasil
}

\begin{abstract}
Resumo
Tendo como base os desafios envolvidos na produção oral (LEVELT, 1989), especialmente na L2 (KORMOS, 2014), este estudo examina o planejamento estratégico (ELLIS, 2005) e seu impacto no desempenho de aprendizes de inglês como L2 em uma tarefa oral,por eles planejada, de forma individual e/ou colaborativa. Com o aporte da hipótese da produção (SWAIN, 1985), esta pesquisa analisou aspectos quantitativos e qualitativos das narrativas produzidas por 17 adolescentes do ensino médio. No que diz respeito à análise quantitativa, houve uma vantagem estatisticamente significativa para a variável outcome quando os participantes planejaram colaborativamente. Na mesma direção, fluência e acurácia também apresentaram resultados favoráveis a essa condição de planejamento, apesar de esses não serem estatisticamente significativos. Os dados qualitativos, por sua vez, apontaram uma grande aceitação das tarefas orais com o uso do WhatsApp. Em vista dos resultados indicados, sugerese a adoção de tarefas que incluam o planejamento estratégico a fim de diminuir as demandas atencionais na produção oral em L2 (FORTKAMP, 2000) como recurso pedagógico atraente para auxiliar aprendizes de inglês no desenvolvimento da língua de forma contextualizada.

Palavras-chave: Tarefa Oral; Planejamento Estratégico; Trabalho Colaborativo; Hipótese da Produção; Aquisição de L2; WhatsApp.
\end{abstract}

\footnotetext{
"Mestre em Inglês: estudos linguísticos e literários pela Universidade Federal de Santa Catarina. Seus interesses de pesquisa incluem aprendizagem colaborativa, produção oral em L2, produção escrita em L2, autonomia na aprendizagem e o ensino embasado em tarefas. Seu e-mail é rafaelzaccaron@gmail.com. ORCID: http://orcid. org/0000-0001-7796-501X.

${ }^{* *}$ Tem bacharelado, mestrado e doutorado na área de ensino/aprendizagem de língua estrangeira pela Universidade Federal de Santa Catarina. É docente no Centro de Comunicação e Expressão - Departamento de Língua e Literatura Estrangeiras na área de Inglês dessa mesma instituição. Seus interesses de pesquisa incluem consciência metalinguística, foco na forma, aprendizagem e escrita colaborativa, produção oral em língua estrangeira e o ensino embasado em tarefas. Seu e-mail é donescax@gmail.com. ORCID: https://orcid. org/0000-0002-2560-2919.

${ }^{* * *}$ Mestre (1983) e doutora (2006), em Letras/Lingüística Aplicada pela UFSC, é professora dessa instituição desde 2010. Tem experiência em Linguística Aplicada, cujos temas de interesse são: ensino e aprendizagem de L2 numa perspectiva cognitiva, com foco no Ensino Baseado em tarefas para ambientes instrucionais e virtuais, e formação de professores. Seu e-mail é raqueldely@gmail.com. ORCID: https://orcid.org/0000-00032851-1868.
} 
"HANG ON A SEC, TEACHER": COLLABORATIVE AND INDIVIDUAL STRATEGIC PLANNING AS A MEANS TO PERFORM L2 ORAL TASKS AT A PUBLIC SCHOOL Abstract

Bearingin mind the challenges involved in speech production (LEVELT, 1989), especially in the L2 (KORMOS, 2014), this study examines strategic planning (ELLIS, 2005) and its impact on the performance of learners of English as an L2 in an oral task carried out after they had planned it individually or collaboratively. Drawing from the output hypothesis (SWAIN, 1985), this piece of research analysed quantitative and qualitative data from the narratives produced by 17 high school students. The quantitative analysis showed a statistically significant advantage for the outcome variable when the participants planned collaboratively. At the same time, while the results for fluency and accuracy were not statistically significant, they also favoured the same planning condition. The qualitative data indicated wide acceptance of oral tasks with the use of WhatsApp. It is suggested that the adoption of tasks that include strategic planning as a means to lessen the attentional burden involved in $\mathrm{L} 2$ speech production (FORTKAMP, 2000) may be an attractive pedagogical resource to help English students to learn the language in a contextualized way.

Keywords: Oral Task; Strategic Planning; Collaborative Work; Output Hypothesis; Second Language Acquisition; WhatsApp. 


\section{Introdução}

Quando a proposta da investigação que originou este trabalho foi apresentada ao professor responsável por pesquisas no colégio cuja coletafoi realizada, sua primeira observação foi: "mas você sabe que os alunos não estão acostumados a planejar para tarefas". De fato, apesar de muitas pesquisas no campo do ensino baseado em tarefas (D'ELY; MOTA; BYGATE, 2019; FOSTER; SKEHAN, 1996; GUARÁ-TAVARES, 2016; ORTEGA, 1999, 2005; PANG; SKEHAN, 2014; SPECHT, 2017; SPECHT; D’ELY, 2017; XHAFAJ; MUCK; D’ELY, 2011; XHAFAH, 2013) apresentarem dados que corroboram esse planejamento como ferramenta benéfica para a performance de aprendizes de segunda língua (L2 ${ }^{1}$ ), alunos da escola pública no Brasil, de forma geral, não têm exposição a tal recurso pedagógico para realização de tarefas nas aulas de L2.

Considerando o ensino baseado em tarefas como proposta pedagógica para o ensino de L2 - no qual, mesmo oportunizando aos aprendizes momentos de "foco na forma" (LONG, 2015), dá-se destaque para o uso de língua com fins comunicativos -, o planejamento estratégico como pré-tarefa (SKEHAN, 1996) surge como um momento em que a carga cognitiva da tarefa pode ser balanceada.

Tal proposta, elaboradapor Skehan $(1996,1998)$ com base na teoria do processamento da informação (MCLAUGHLIN, 1987), postula que em função de nossos recursos atencionais serem limitados, e da função primordial da linguagem ser comunicar uma dada mensagem, não podemos esperar que aprendizes de L2 destinem atenção para a acurácia com a qual produzem uma dada mensagem, ou mesmo que tentem variar seu vocabulário, ou utilizar estruturas sintáticas mais complexas; isso porque a atenção desses falantesestá focada no significado e na rapidez com a quala mensagem pode ser produzida.

Ao observar o argumento de Skehan $(1996,1998)$, um leigo poderia entender que utilizamos pouco da nossa "capacidade cerebral". Todavia, o fato é que a produção da fala, que nos parece tão natural, fácil, e fluida, envolve processos complexos e só acontece tão facilmente na nossa língua materna graças a alguns desses processos serem automatizados - isto é, demandam pouca (ou nenhuma) atenção - (LEVELT, 1989). Por isso, quando pensamos nesses mesmos processos - de conceitualização, formulação, articulação, e monitoramento - na produção de L2, os desafios ficam claros, pois, enquanto na nossa língua materna a atenção é necessária basicamente para decidir o que comunicar, como expressar a intenção comunicativa (conceitualização) e para o monitoramento, na L2 precisamos, também, nos ater aos processos de formulação (produção das estruturas gramaticais e fonológicas da mensagem) e de articulação (transformação do discurso interno em externo através do controle dos músculos articulatórios responsáveis pela execução dos sons) da fala (KORMOS, 2014; LEVELT, 1989). Além disso, na produção oral em L2 é preciso lidar com o fato de que i) há pelo menos uma outra língua ali estabelecida, com um léxico provavelmente muito mais completo e que compete com itens lexicais da L2;ii) o conhecimento da sintaxe da L2 é incompleto; e iii) o aparelho 
fonador, ao menos inicialmente, conta apenas com planos articulatórios para sílabas da língua materna (KORMOS, 2014).

Desse modo, ao percebermos a demanda atencional que a fala na L2 implica, faz muito sentido Skehan $(1996,1998)$ propor que aprendizes tenham maior chance de produzir a L2 com mais acurácia e fluência se tiverem um tempo anterior ao momento da fala para a conceitualização da mensagem a ser produzida; tanto no nível macro - o das ideias- quanto no nívelmicro - as palavras e as estruturas linguísticas necessárias para que a mensagem seja realizada. Ademais, dependendo da maneira como a tarefa é proposta, há a possibilidade de que esses aprendizes possam se arriscar a usar formas novas em seu repertório linguístico (SKEHAN, 1996, 1998) que irão lhes permitir testar hipóteses sobre a língua e, talvez, levar à reestruturação (SWAIN, 2000).

Por ter esse olhar que vê a oportunidade para planejamento estratégico prétarefa como benéfica não apenas para a produção oral imediata, mas também como possibilidade de levar o aprendiz a expandir e consolidar seu conhecimento da L2, este estudo está interessado nas discussões acerca do trabalho colaborativo para a produção oral em L2. A esse respeito, nas últimas três décadas, uma série de estudos de viés interacionista baseados em tarefas (BENISS; BAZZAZ, 2014; KOWAL; SWAIN, 1994; STORCH, 2002; SWAIN, 2000; SWAIN e LAPKIN, 2005; XHAFAJet al., 2011; XHAFAH, 2013) tem ressaltado o papel fundamental da interação para produção em L2. No entanto, a grande maioria da produção que enfatiza a aquisição de L2 baseada em tarefas - incluindo os supracitados teve adultos como participantes.

Dessa forma, tendo em vista a falta de trabalhos sobre a população adolescente em ambiente escolar, e ciente das discussões teóricas do campo, este estudo teve por objetivo principal analisar o impacto do planejamento estratégico individual e em grupos na realização de uma tarefa oral por aprendizes adolescentes de inglês como L2. Ainda, como objetivossecundários, pretendeu-se investigar, de forma experimental, o potencial pedagógico do uso de tarefas no ensino de L2 (TAVAKOLI; FOSTER, 2008) na escola pública e a aceitação do WhatsApp como ferramenta para a realização de tarefas.

Para o cumprimento desses itens, foram analisados dados quantitativos e qualitativos advindos do planejamento para a realização das tarefas e das mensagens gravadas em língua inglesa (tarefa em si) por 17 alunosda segunda série do ensino Médio em uma escola pública, estudantes dessa língua como L2. Com tal movimento pode-se contribuir para o campo pedagógico em relação à elaboração e implementação de tarefas orais em L2 por professores cujo objetivo é a promoção do desenvolvimento da habilidade oral.

No que diz respeito à inclusão de tarefas na aula de L2, o processo pode ocorrer inicialmente de forma pontual, como nessa pesquisa, com vistas a se tornar um processo gradual, conforme o professor observa os resultados e a aceitação por parte dos alunos. Nesse contexto, o planejamento estratégico para a tarefa oferece recursos que visam diminuir as demandas de processamento envolvidas na produção oral em L2 (KORMOS, 2014), de maneira que, mesmo 
não dominando todos os aspectos linguísticos da L2, ao planejar, o aprendiz recorra a processos estratégicos internos (de forma individual) e externos (com a ajuda de colegas) de modo a suprir lacunas de conhecimento na L2.Dessa forma, este estudo também tem como objetivo contribuir para o campo da psicolinguística, uma vez que nos permite especular como o processamento oral na L2 pode acontecer de forma diferente quando há tempo para o planejamento da fala e a possibilidade depodercontar com o apoio de colegas.

\section{Fundamentação Teórica}

\section{1 Planejamento Estratégico para a Produção de Tarefas Orais}

De acordo com o framework de Skehan (1996), toda tarefa deve ser precedida de uma pré-tarefa, dado que essa etapa objetivaatenuar as fortes demandas deprocessos cognitivos complexos que precedem sua execução; como i) aqueles que implicam a decisão de o que e como comunicar a mensagem; ii) a familiaridade/ não familiaridadedo aprendiz com o tipo de tarefa, fator imprescindívelpara o bom desempenho, que, por seu turno, pode sofrer impactos de acordo com as características da tarefa (o quão estrutural ela é, número de elementos que possui, recursos visuais que possa ter etc.); iii) a existência/ não existência de pressão de tempo e o tipo de negociação e mediação durante sua execução (Skehan, 1996, 2015). Cabe salientarque a prétarefa possibilita a manipulação prévia do insumo na L2 (input) por professores ou pesquisadores e, por isso, tem sido o objeto de estudo de diversas pesquisas (XHAFAJet al., 2011). Ainda, dentre as inúmeras possibilidades de uso da prétarefa, o planejamento estratégico firmou-se como ferramenta de destaque em diversos estudos empíricos (D’ELY; MOTA; BYGATE, 2019).

O planejamento estratégico é o tempo dado para o aprendiz organizar a tarefa (ELLIS, 2005), uma vez que há a possibilidade de se planejar o que e como falar, conhecendo-se as instruções e os materiais disponibilizados. Logo, é uma ação metacognitiva ${ }^{2}$ (ANDERSON, 2002; OXFORD, 2013) com vistas a facilitar o processamento da linguagem, que, conforme já citado, é complexo e demanda tempo e atenção (KORMOS, 2014; LEVELT, 1989), sendo esse um recurso limitado, seletivoe necessário para a aprendizagem (SCHMIDT, 2001).

Ainda sobre a atenção, é pertinente a consideração de que o sucesso na aprendizagem de uma segunda língua vai além do que está presente no insumo (ORTEGA, 2009). Éindispensável que aspectos da linguagem possam ser percebidos $\left(\right.$ noticed $\left.^{3}\right)$ e, portanto, a atenção é fundamental para o planejamento estratégico. Esse tipo específico de planejamento está também fundamentado na ideia de foco-na-forma, um conceito cunhado por Long (1991, 2010, 2015), que afirma que a atenção aos elementos linguísticos pode acontecer durante a execução de uma tarefa cujo foco principal é o significado. Assim, esse planejamento para executar uma tarefa, capaz de levar o aluno a prestar atenção na forma, tem atraído o interesse de pesquisadores e profissionais da área. 
Os resultados de estudos empíricos acerca do planejamento estratégico para tarefas orais (D'ELY; MOTA; BYGATE, 2019; FOSTER; SKEHAN, 1996; GUARÁ-TAVARES, 2009; LI; CHEN; SUN, 2015; MEHNERT, 1998; PANG; SKEHAN, 2014; SKEHAN; XIAOYUE; QIAN; WANG, 2012; SPECHT, 2017; SPECHT; D'ELY, 2017; XHAFAJet al., 2011; XHAFAH, 2013), em geral, apontam para o impacto positivo dessa condição em diferentes dimensões da performance, sendo elas: complexidade, acurácia e fluência ${ }^{4}$ (doravante CAF). Além disso, os resultados desses estudos indicamalgumas direções, a saber: i) o tempo de 10 minutos para o planejamento mostrou-se adequado em Mehnert (1998) e tal intervalo firmou-se como referência em pesquisas posteriores; ii) em um estudo seminal para a área, Foster e Skehan (1996) analisaram o impacto do planejamento e as características da tarefa em relação à CAF e os resultados apontaram, emlinhas gerais, que, ao direcionar a atenção para determinado(s) aspecto(s) da competência oral, a produção do aprendiz acaba penalizando outras esferas. Tal efeito, denominado trade-off pelos autores, é frequentemente apontado como uma possível explicação para resultados de estudos nos quais, por mais que a tarefa tenha sido manipulada para diminuir demandas atencionais, não foi possível perceber melhora em todos os três aspectos (complexidade, fluência, e acurácia) na fala dos participantes (GUARÁ-TAVARES, 2009; MEHNERT, 1998; SKEHAN et al., 2012; SPECHT, 2017; XHAFAH, 2013). Essas tendências mostram-se válidas também para o planejamento em grupo.

\subsection{Trabalho Colaborativo em Grupo e a Hipótese da Produção}

O interesse sobre o trabalho colaborativo e seu impacto na aprendizagem de L2 vem crescendo no Brasil, em especial desde os anos de 1980; contudo, sua mobilização no campo de ensino baseado em tarefas é fato mais recente. Na presente pesquisa, a análise dessa condição de trabalho é ancorada na hipótese da produção de Swain (1985, 2001; SWAIN; LAPKIN, 2005), que observou estudantes de L2 no Canadá e verificou que mesmo a rica exposição ao insumo em inglês não garantia uma proficiência elevada na língua. Levando isso em conta, de acordo com o autora, para que a aquisição de L2 ocorra, é necessário que aprendizes manipulem elementos linguísticos por meio de diálogos colaborativos (LIMA; COSTA, 2010; SWAIN, 2000). Partindo dessa interação, o aprendiz pode vir a prestar atenção nas suas lacunas linguísticas e buscar a adequação para sua produção. Nessa perspectiva, é oportuno destacar que a necessidade de tornar o insumo compreensível ao interlocutor pode levar aprendizes a focar em aspectos estruturais da língua de forma contextualizada, uma vez que tal necessidade emerge durante a interação (LIMA; COSTA, 2010).

Segundo Swain (2000), a hipótese da produção para a aquisição de L2 está ancorada em três funções: i) a percepção (noticingthe gap) que ocorre quando o aprendiz identifica lacunas no seu conhecimento linguístico na L2 e dessa forma busca soluções para preenchê-las; ii) a formulação e verificação de hipóteses, pois o aprendiz recorre a recursos internos (insumo) e externos (interlocutor) 
ao reconhecer problemas na comunicação, a fim de preencher a(s) lacuna(s) linguísticas, testando hipóteses para solucionar o problema); e iii) a metalinguagem, já que o aprendiz pode se voltar para as relações entre forma e função de maneira contextualizada - extrapolando o aspecto semântico, incluindo a sintaxe (SWAIN, 2000), ao refletir sobre o uso da língua e suas dificuldades linguísticas através de metatalk(SWAIN, 2001) - discussão de aspectos linguísticos concernentes às lacunas no conhecimento linguístico da L2. Por questões inerentes às funções da hipótese de Swain, tais quais a atenção, o foco na forma, e o uso de metalinguagem, é possível tecer uma relação com o planejamento estratégico.

É importante ressaltar que ao focar no papel da produção em sua hipótese, Swain (1985) não diminui a importância que o insumo tem para aquisição de uma L2; pelo contrário, o acesso a insumo compreensível é parte do processo que leva à produção. Entretanto, a autorarefuta a noção de que a produção é meramente uma consequência da aprendizagem de uma L2, como ressaltam Loewen e Sato (2018). Para que a aprendizagem ocorraé necessário haver a produção "forçada", 5 uma vez que aprendizes tendem a focar sua atenção em aspectos semânticos ao processar o insumo (SWAIN, 1985). Por outro lado, a produção gera a necessidade de o aprendiz perceber (notice) aspectos linguísticos funcionais que estão atrelados ao significado da mensagem, seja ela oral ou escrita. Finalmente, Izumi (2003) ressalta que a produção tem um papel importante no modelo de processamento de fala de Levelt através do monitoramento da fala desde o momento da conceitualização, dado que, "a produção desencadeia uma série de processos psicolinguísticos que são benéficos ao aprendizado de língua" (IZUMI, 2003, p. 187). Dentre esses processos estão a codificação gramatical e o monitoramento, que fazem com que o aprendiz interaja com o meio externo, usando atenção seletiva ao insumo, com o objetivo de achar uma solução linguística para sua produção (IZUMI, 2003).

Com relação aos agrupamentos para interação entre aprendizes, Hyde (1993) fez um estudo qualitativo cujos partícipes demonstraram a preferência pelo trabalho em grupos em detrimento do individual ou em pares, sendo essa última condição a menos preferida. Ao justificar tal posicionamento, os participantes ressaltaram que quando o aprendiz não se sente confortável em produzir em dupla com o colega, tal trabalho não é possível, fato esse que pode ser amenizado ao se reunirem em grupo. Do ponto de vista pedagógico, o trabalho em grupo muda a dinâmica da interação na sala de aula, visto que aumenta o tempo de fala do aprendiz e pode elevar a motivação da prática oral, pois configura um ambiente mais seguro para a fala em comparação à exposição para toda a sala de aula (RICHARDS; FARRELL, 2011). Por essa razão, grupos de quatro aprendizes são mais adequados para o controle da sala de aula. Logo, este estudo parte da premissa de que o planejamento colaborativo em grupos pode diferir dos resultados obtidos em estudos semelhantes nos quais os participantes trabalharam em pares.

Considerando a pluralidade de estudos que têm investigado o trabalho colaborativo, é possível observar algumas constantes. Essa opção parece 
contribuir para que aprendizes notem lacunas no seu conhecimento (KOWAL; SWAIN, 1994; LIMA; COSTA, 2010; STORCH, 2002). Ademais, a performance pode sofrer um impacto positivo do trabalho colaborativo (BENISS; BAZZAZ, 2014); porém, taldiferença pode não se mostrar estatisticamente significativa quando aprendizes planejam em pares (XHAFAJ, 2013). Ao mesmo tempo, algumas desvantagens também foram relatadas, como a ansiedade gerada pelo trabalho em grupo (BATSTONE, 2012) e a desconsideração de sugestões causada por diferenças acentuadas do nível de proficiência (LYNCH; MACLEAN, 2000; STORCH, 2002) entre os aprendizes. Ainda em relação a possíveis obstáculos, sublinha-se o fato de que colocar aprendizes para trabalharem em grupo não significa que a ação se dará de forma colaborativa (PAIVA, 2015).

Como pode ser observado nessa breve revisão de literatura, embora haja muitos estudos que investigaram a produção de tarefas orais em L2, poucos focaram o planejamento individual versus planejamento colaborativo (BALEGHIZADEH, 2010; FOSTER; OHTA, 2005; STORCH, 2002;XHAFAJet al., 2011; XHAFAH, 2013). Nesse último grupo, a maioria comparou o desempenho na tarefa oral realizada após o planejamento individual versus o planejamento em pares, como observado em Xhafajet al. (2011) e Xhafaj (2013). Nesse cenário, considerando que o contexto brasileiro do ensino de L2, especialmente o da escola pública, envolve muitas vezes salas de aula com muitos alunos, a variedade nas formas de agrupamento é necessária. Reitera-se com isso a questão de queinvestigações acerca do impacto do planejamento colaborativo em grupo ${ }^{6}$ é pertinente e, por extensão, a decisão de analisar o trabalho em grupo num contexto escolar com adolescentes colabora para o preenchimento da supracitadalacuna da área do ensino baseado em tarefas.

A partir dessas considerações e do aporte teórico apresentado, o objetivo deste trabalho é analisar o impacto do planejamento estratégico individual e em grupos para a realização de uma tarefa oral por aprendizes adolescentes de inglês como L2. A fim de alcançar tal objetivo, as seguintes perguntas de pesquisa (PP) e hipótese $(\mathrm{H})$ foram formuladas:

PP 1 - O desempenho oral do grupo de planejamento colaborativo é estatisticamente mais fluente, gramaticalmente preciso e adequado do que o desempenho do grupo de planejamento individual?

$\mathrm{H}$ - O desempenho oral dos estudantes após planejarem em grupo é mais fluente, gramaticalmente preciso e apropriado do que o desempenho oral desses alunos após planejarem individualmente?

PP 2 - Qual a percepção dos alunos participantes sobre as diferentes condições de performance?

A próxima seção apresenta o método empregado na pesquisa. A seguir, serão detalhados os critérios para a seleção dos participantes, o contexto da pesquisa, bem como os procedimentos tanto para a coleta quanto para a análise dos dados. 


\section{Método}

O presente estudo caracteriza-se por uma abordagem mista (DÖRNYEI, 2007), com dados quantitativos e qualitativos. Nessa investigação, a produção oral em L2 foi analisada em relação à fluência, aos desvios da norma padrão (acurácia) e a uma terceira variável de viés pragmático (outcome), que diz respeito a quão efetiva a narrativa se mostrou em cumprir o objetivo estabelecido pela tarefa; essa análise teve como objetivo identificar se a diferença no desempenho oral obtido após as duas formas de planejamento pesquisadas - individual e em grupo - foi estatisticamente significativa. Já o aspecto qualitativo da pesquisa refere-se à percepção dos participantes em relação ao processo de planejamento, à produção da tarefa oral e ao uso do WhatsApp.

\subsection{Contexto pedagógico e participantes}

Participaram da investigação 17 alunos, com idades entre 15 e 17 anos ( $m$ = 16,11 anos), matriculados no ensino médio de um colégio de ensino regular, mantido por uma universidade federal como colégio de aplicação ${ }^{7}$ (doravante CA). A fim de traçar o perfil de aprendiz de inglês como L2 desses participantes, um questionário formulado pelos pesquisadores foi aplicado. Segundo as respostas obtidas, a maior parte dos participantes no momento da coleta: (a) não tinha estudado inglês fora da escola, (b) nem morado ou visitado um país onde inglês é a L1 e, em grande parte, (c) tinha entre 1 e 2 horas de contato semanal com a língua inglesa fora do CA. Em relação a esse contato com a L2 fora da sala de aula, ele se dava nos seguintes contextos (ordem decrescente de menções): i) ouvindo, traduzindo e cantando músicas; ii) escrevendo / lendo na internet; iii) jogando games; iv) usando aplicativos de ensino de língua (e.g., Duolingo); v) falando com amigos; vi) assistindo a filmes ou séries; e vii) falando com pais ou familiares.

Como procedimento prévio, as tarefas da pesquisa foram detalhadas para a professora de inglês do CA, que também foi alertada sobre a necessidade de a pesquisa ser desenvolvida com uma turma que estivesse, de modo geral, em um nível intermediário da L2. Dessa forma, a escolha da turma (segunda série do ensino médio) foi feita com base na percepção da docente que acompanhou esses estudantes no ano anteriore na expectativa de que a exposição a aulas de inglês por cinco anos tivesse desenvolvido a habilidade em nível similar ao nível intermediário (B1 no Common European Framework ofReference) na L2. ${ }^{8}$ Como a proficiência avançada na L2 foi apontada como um inibidor para a negociação de significado no planejamento em pares (XHAFAJ et al., 2011), optou-se por uma série em que se esperava encontrar a maioria dos alunos com nível intermediário na L2. Quanto à proficiência, ressalta-se que não foi medida, dado que essa variável, apesar de impactar nos resultados, não era fator para a exclusão de participantes e, mais do que isso, a heterogeneidade de proficiência da população estudada foi proposital, a fim de refletir a diversidade encontrada em sala de aula de L2, não só da escola pública. 


\subsection{Instrumentos e Coleta de Dados}

Para o cumprimento da investigação, os seguintes instrumentos foram utilizados: i) um questionário do perfil de aprendiz de inglês como L2;ii) duas tarefas orais monológicas;iii) as gravações em áudio das interações em grupo durante o planejamento; e iv) um questionário de percepção da tarefa. Esses instrumentos foram previamente testados em um estudo piloto ${ }^{9}$ conduzido em outra escola pública.

Duas tarefas orais monológicas, vistas como estímulos semelhantes para a produção oral em L2, de acordo com o estudo piloto, foram utilizadas a fim de possibilitar que cada participante planejasse a tarefa sob as duas formas de planejamento foco deste estudo (colaborativo e individual) sem repetir a mesma tarefa, tendo em vista que estudos indicam o impacto da repetição (BEI, 2013; BYGATE, 2001;LYNCH; MACLEAN, 2000; ZACCARON, 2018b) na performance. A primeira delas (tarefa $A$, ver na sequência) foi baseada no estudo de Mehnert (1998). A segunda (tarefa B, ver na sequência) foi criada porXhafaj et al. (2011) com o objetivo de que fosse similar à elaborada porMehnert. Ambas as tarefas mostraram aceitação positiva pelos participantes em Xhafaj et al. (2011) e Xhafaj (2013). Por essa razão, dado o histórico positivo em pesquisas anteriores, as tarefas foram adaptadas para o presente estudo, sendo a modificação mais marcante a introdução do uso do celular pelos participantes; assim, a gravação foi realizada via áudio do WhatsApp.

A escolha pelo WhatsApp como instrumento de pesquisa é reflexo da intensa relação entre tecnologia e ensino, sendo que, atualmente, por ser um aplicativo popular entre os aprendizes, seu uso em sala de aula pode diminuir o impacto da ansiedade gerada pela demanda da produção oral (HAN; KESKIN, 2016). Além do que, o uso de WhatsApp entre professores e alunos é relativamente comum para fins pedagógicos (WEISSHEIMER; CALDAS; MARQUES, 2018). Ainda em relação à validação do WhatsApp como ferramenta para a coleta de dados, ressalta-se que os dados provenientes do questionário de perfil indicaram que os participantes faziam uso de tal ferramenta. Abaixo são apresentadas as tarefas.

Quadro 1 - Tarefa A

\section{Trabalho em grupo:}

Você marcou para encontrar seus colegas de sala pra fazer um trabalho em grupo. Como estava chovendo você ficou com preguiça de sair da cama e não foi. Mas agora você precisa ligar pra eles e dar uma boa desculpa. Invente uma história pra justificar não ter ido encontrar eles. Sua desculpa deve ter as seguintes palavras: house, toget e umbrella (em qualquer ordem). Você vai deixar uma mensagem de voz (áudio) no whats de um(a) colega do grupo. A duração você decide. Adicione o número (XX) XXXX-XXXX nos seus contatos, esse é o número para o qual você deve enviar a mensagem.

Fonte: adaptado deXhafaj (2013). Zaccaron (2018a). 
Quadro 2 - Tarefa B

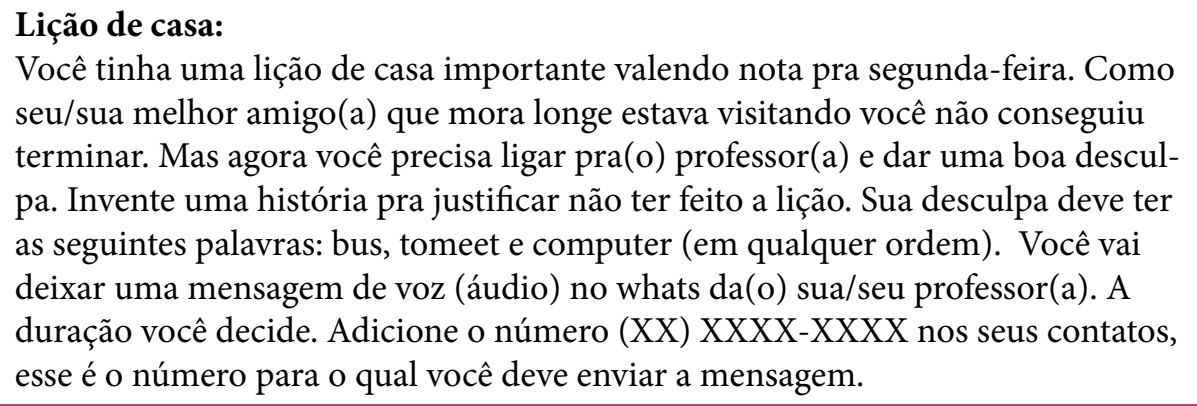

Você tinha uma lição de casa importante valendo nota pra segunda-feira. Como seu/sua melhor amigo(a) que mora longe estava visitando você não conseguiu terminar. Mas agora você precisa ligar pra(o) professor(a) e dar uma boa desculpa. Invente uma história pra justificar não ter feito a lição. Sua desculpa deve ter as seguintes palavras: bus, tomeet e computer (em qualquer ordem). Você vai deixar uma mensagem de voz (áudio) no whats da(o) sua/seu professor(a). A duração você decide. Adicione o número (XX) XXXX-XXXX nos seus contatos, esse é o número para o qual você deve enviar a mensagem.

Fonte: adaptado de Xhafaj (2013). Zaccaron (2018a).

Após a observação de oito aulas da turma, realizadas pelo primeiro autor, os alunos foram convidados a participar da pesquisa e, por serem menores de idade, o TCLE - Termo de Consentimento Livre e Esclarecido - foi assinado por eles e por seus pais ou responsáveis. Os procedimentos apresentados na sequência foram baseados em Xhafaj (2013).

No dia da coleta de dados, a turma foi dividida em duas, em salas separadas, sendo que cada participante recebeu uma folha com as instruções para a tarefa, uma folha com dicas de planejamento e uma folha em branco para uso durante o planejamento;as instruções também foram dadas de forma oral. Em uma sala, oito alunos (quatro tarefas $\mathrm{A}+$ quatro tarefas B) planejaram individualmente por 12 minutos. $^{10}$ Ao mesmo tempo, em uma segunda sala, novealunos (um trio tarefa $\mathrm{A}+$ dois trios tarefa $\mathrm{B}$ ) planejaram em grupos previamente definidos pela professora de inglês da classe, pelo mesmo intervalo de tempo, sendo que a interação de cada grupo foi gravada por um gravador de voz digital Sony IcdPx440. Diferentemente de pesquisas similares (XHAFAJ et al., 2011; XHAFAH, 2013), cujo planejamento usando a L2 foi obrigatório, o presente estudo, tendo o contexto e participantes em mente, não só permitiu, como incentivou que o planejamento ocorresse usando a L1, levando em consideração que discussões sobre metalinguagem somente na L2 poderiam excluir alguns participantes do benefício proporcionado por esse processo(VIDAL, 2010). Esgotados os 12 minutos, foi solicitado a todos que pegassem seus celulares, ${ }^{11}$ deixassem os papéis na mesa e saíssem da sala procurando um lugar no corredor,distantes uns dos outros,para gravar a mensagem individualmente uma única vez, sem repetir a gravação (a fim de evitar efeitos de repetição e prática). Finalmente, terminadas as gravações (e após elas terem sido enviadas por WhatsApp para o celular do pesquisador responsável pela coleta de dados), cada participante retornou à sala e preencheu individualmente o questionário de percepção sobre a tarefa. Esse processo foi supervisionado pelos assistentes.

O processo descrito no parágrafo anterior tomou o tempo de uma aula de inglês no CA (40 minutos). No dia da coleta houve o intervalo da escola, de 20 minutos, entre as aulas. Na sequência, foi solicitado aos estudantes que trocassem de sala para a segunda aula de inglês (aula-faixa). A troca de sala, no segundo momento, envolveu a alternância da tarefa e da condição de planejamento. Por 
exemplo: uma aluna, que planejou individualmente para realizar a tarefa $\mathrm{B}$ no primeiro momento, planejou em grupo a realização da tarefa A na segunda etapa da coleta. Esse procedimento foi adotado com o intuito de amenizar efeitos da repetição (BYGATE, 2001) na performance.

O questionário de percepção da tarefa teve como objetivo capturar a reação dos participantes em relação aos diversos aspectos envolvidos na tarefa. Entre eles: a satisfação em relação às tarefas, a ter tido tempo para planejar, ao tempo dado para o planejamento, à condição de planejamento (individual ou em grupos) e ao uso dos celulares para gravar as mensagens. A maioria das perguntas (e.g., "Quanto você gostou de gravar a mensagem usando o celular?") apresentava quatro expressões faciais (ver figura 1), dentre as quais os participantes assinalavam aquela que correspondia ao seu sentimento em relação à pergunta.

Figura 1 - Expressões faciais do questionário
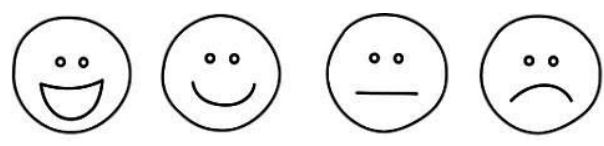

Fonte: elaborada pelosautores (2019).

\subsection{Análise dos dados}

Como mencionado, esta pesquisa apresenta dados quantitativos e qualitativos. Para a análise dos dados quantitativos, foram utilizados o programa de estatística SPPS 17.0 e o Excel 2010. Esses dados provêm das mensagens de áudio de WhatsApp, gravadas pelos participantes, que foram transcritas para a análise de três medidas de produção oral em L2: i) fluência, ii) precisão gramatical (acurácia) e iii) adequação (outcome). Uma breve explanação sobre os critérios de cada medida é apresentada na sequência.

Com o propósito de medir a fluência e a precisão gramatical, seguindo parâmetros de estudos da área (FOSTER; SKEHAN, 1996), as mensagens orais foram segmentadas em AS-Units. ${ }^{12} \mathrm{~A}$ escolha pela unidade AS-Unit justifica-se pelo aumento do seu uso em pesquisas recentes (BENISS; BAZZAZ, 2014; SPECHT, 2017). Em relação às três medidas de produção oral que constituem as variáveis dependentes do estudo, a operacionalização se deu conforme descrito abaixo.

1 - Fluência: A medida de fluência adotada foi o speech rate unprunned (SRU). Essa medida é calculada através da divisão do número total de unidades semânticas, incluindo repetições e autorreparos, pelo tempo da mensagem em segundos. Multiplica-se esse valor por 60 para obter o número de palavras por minuto.

2 - Precisão gramatical (acurácia): essa medida foi adotada tendo em vista que o presente estudo está inserido na área de aquisição de L2 e, nesse contexto, é importante identificar e mostrar ao aprendiz desvios da norma 
padrão (MICHEL,2017). Três avaliadores ${ }_{1}^{13}$ sendo um falante nativo, analisaram transcrições e áudio das mensagens e indicaram os desvios da norma. Uma vez que os três concordaram com os desvios da norma de cada mensagem, o valor da precisão gramatical foi calculado por meio da divisão do número de desvios da norma pelo número de AS-Units.

3 -Outcome: seguindo uma recente corrente da área, que ressaltou a necessidade do estabelecimento de uma medida pragmática para análise da produção oral em L2 (MICHEL, 2017; PALOTTI, 2009), visto que uma mensagem pode ser fluente e gramaticalmente precisa e ainda assim não alcançar seu objetivo comunicativo, adotou-se a medida outcome, desenvolvida por Farias (2014). Assim, uma tabela para avaliação, contendo cinco aspectos pragmáticos da mensagem, foi apresentada para três avaliadores - sendo novamente um falante nativo - que analisaram transcrições e áudio das mensagens e aferiram uma nota de 1 (muito fraca) a 6 (excelente) a cada uma. Com os dados foi rodado o teste de confiabilidade dos avaliadores e o valor do Cronbach alpha de 0.943 indicou um alto grau de confiabilidade na avaliação individual de cada avaliador. Finalmente, o valor de outcome de cada mensagem foi obtido através da média aritmética das notas dadas por cada um dos avaliadores.

Como a pergunta de pesquisa 1 visa comparar a performance dos mesmos participantes sob duas condições distintas (planejamento individual e planejamento em grupo), o teste paramétrico $\mathrm{T}$ de amostras pareadasfoi escolhido, tendo, também, em vista que os dados apresentaram distribuição normal segundo a análise dos resultados do teste Kolmogorov-Smirnov, valores para assimetria, curtose e histogramas (para esses detalhes, ver ZACCARON, 2018a).

Em relação aos dados colhidos com a finalidade de responder à pergunta de pesquisa 2, sobre a percepção dos participantes, os mesmos foram agrupados em tabelas e analisados de forma qualitativa. Também de forma qualitativa foram analisadas as gravações das interações dos alunos com o propósito de dar suporte à resposta da pergunta 1 .

\section{Resultados e Discussão}

\subsection{Dados quantitativos}

Durante a fase de transcrição, observou-se que algumas mensagens, sete do total de 34, continham algumas palavras em português, apesar de as instruções orais e escritas para a tarefa requisitarem o uso exclusivo da L2 para a mensagem. Tendo em vista o caráter ecológico da pesquisa e o contexto pesquisado (escola pública), tais mensagens foram consideradas para a análise quantitativa. No entanto, uma vez que o resultado na comparação das medidas seria afetado, o seguinte critério foi adotado para o uso de L1 nas mensagens: quando a unidade de AS-Unit continha uma palavra em português, o desvio à norma em acurácia 
foi contabilizado como dois desvios, quando a unidade de AS-Unit continha mais de 1 palavra em português a mesma foi descartada, como no exemplo a seguir, que não foi contabilizado nos resultados quantitativos:

"I não posso sair house"

Quanto aos dados quantitativos, foi possível observar os seguintes resultados na tabela 1, que mostra a estatística descritiva. Os valores das três variáveis dependentes - fluência, acurácia e outcome - são comparados em relação à variável independente - condição de planejamento -, de modo a verificar possíveis diferenças.

Tabela 1- Estatística Descritiva - planejamento individual e planejamento colaborativo

\begin{tabular}{llllll}
\hline Medida & $\begin{array}{l}\text { Condição de } \\
\text { planejamento }\end{array}$ & Min & Max & M & DP \\
\hline \multirow{2}{*}{ Fluência } & Individual & 43,30 & 198,60 & 109,87 & 41,10 \\
& Colaborativo & 68,60 & 177,60 & 116,94 & 33,93 \\
\hline \multirow{2}{*}{ Acurácia } & Individual & 0,17 & 2,77 & 1,43 & 0,82 \\
& Colaborativo & 0,17 & 2,33 & 1,23 & 0,69 \\
\hline \multirow{2}{*}{ Outcome } & Individual & 6,30 & 28,60 & 17,79 & 7,12 \\
& Colaborativo & 6,70 & 29,70 & 19,37 & 6,96 \\
\hline
\end{tabular}

Min = valor mínimo; $\mathrm{Max}=$ valor máximo; $\mathrm{M}=$ médias; $\mathrm{DP}$ = desvio padrão; Fluência = SRU;

Acurácia =número de desvios à norma por AS-unit; Outcome = medida pragmática (valor máximo 30).

Fonte: elaborada pelos autores (2019).

Como se vê, embora não haja diferenças marcantes nos valores de médias (M) da tabela 1, o que se pode verificar, com efeito, éuma tendência em favor da variável independente planejamento colaborativo, semelhante ao encontrado por Xhafajet al. (2011). Quando se avalia as variáveis separadamente, é possível notar que (a) a fluência apresenta uma vantagem para o planejamento colaborativo ( $M=$ 116,94), com mensagens em média 6\% mais fluentes em relação ao planejamento individual $(\mathrm{M}=109,87)$; (b) a acurácia da produção após planejamento colaborativo foi superior $(M=1,23)$, com $14 \%$ menos desvios da norma, a da produção após o planejamento individual $(\mathrm{M}=1,43)$, e, finalmente (c) o outcome colaborativo $(\mathrm{M}=19,37)$ apresentou valores $8 \%$ mais altos do que o outcome individual $(\mathrm{M}=$ 17,79). Ainda em relação aos dados da tabela 1, observa-se que os valores de desvio padrão - que indicam os valores de dispersão dos dados em relação à média do grupo - apresentam uma vantagem para o planejamento colaborativo, dadoque essa condição apresenta uma variação menor em tais valores. 
Com o objetivo de analisar se as diferenças entre as médias, expostas na tabela 1, foram estatisticamente significativas, testes $\mathrm{T}$ de amostras pareadas foram rodados para cada medida. A tabela 2 apresenta os valores dos testes.

Tabela 2 - Resultados da performance comparada em pares

\begin{tabular}{l|l|l|l|l}
\hline & \multicolumn{2}{l|}{ Amostra Pareadas } & \multirow{2}{*}{ Sig. (2-tailed) } \\
\cline { 2 - 4 } & Médias & Desvio Padrão & $\begin{array}{l}\text { Média Desvio } \\
\text { Padrão }\end{array}$ & 0,285 \\
\hline Fluência (Ind. - Grupo) & $-7,0$ & 26,3 & 6,4 & 0,148 \\
\hline Acurácia (Ind. - Grupo) & 20,3 & 55,0 & 13,3 & 0,086 \\
\hline Outcome (Ind. - Grupo) & $-1,6$ & 3,5 & 0,8 & \\
\hline
\end{tabular}

Fonte: elaborada pelos autores (2019).

Conforme a tabela 2, os valores de alpha apresentaram-se todos acima de $p>0,05$, de modo que as diferenças entre as duas condições de planejamento não se mostraram significativas. Uma das razões que pode ter influenciado os resultados foi o fato de um grupo de quatro participantes ter usado grande parte do tempo de planejamento para brincar entre si e com o gravador de áudio, segundo as anotações da coleta feitas pelo primeiro autor e os áudios das interações. Sendo assim, os resultados de uma segunda rodada do teste $\mathrm{T}$ de amostras pareadas ${ }^{14}$, excluindo os quatro participantes, são apresentados na tabela $3(\mathrm{~N}=13)$.

Tabela 3 - Resultados da performance comparada em paresWILCOXON

\begin{tabular}{l|l|l|l}
\hline & $\begin{array}{l}\text { FluênciaGrupo - Fluência } \\
\text { - Individual }\end{array}$ & $\begin{array}{l}\text { AcuráciaGrupo - Acurá- } \\
\text { cia Individual }\end{array}$ & $\begin{array}{l}\text { OutcomeGrupo - Out- } \\
\text { come Individual }\end{array}$ \\
\hline Asymp Sig. (2-tailed) & 0,221 & 0,172 & 0,023 \\
\hline
\end{tabular}

Fonte: elaborada pelos autores (2019).

As mudanças nos valores de alpha apresentados na tabela acima novamente não se mostraram estatisticamente significativas para as variáveis fluência e acurácia. No entanto, para a variável outcome o valor de $\mathrm{p}=0,023$ mostrou uma diferença estatisticamente significativa em favor da condição de trabalho colaborativo. Com base nesses dados, a resposta à pergunta de pesquisa 1 - "O desempenho oral do grupo de planejamento colaborativo é mais fluente, gramaticalmente preciso e adequado do que o desempenho do grupo de planejamento individual?" - é: o desempenho oral do grupo de planejamento colaborativo se mostrou mais adequado (outcome) do que o desempenho do grupo de planejamento individual, porém não foi mais fluente e gramaticalmente preciso.

Os valores estatisticamente não significativos para fluência e acurácia foram de encontro à pressuposição de que aprendizes em um contexto escolar de ensino 
médio necessitariam colaborar durante o planejamento e que tal movimento impactaria em todos os aspectos medidos da mensagem oral. Diferentemente do que se vê em Xhafaj et al. (2011), cujos participantes possuíam um alto nível de proficiência na L2, o contexto escolar apresenta, muitas vezes, uma pluralidade maior na proficiência na língua inglesa dentro de uma mesma sala de aula e, por isso, eram esperadas diferenças estatisticamente significativas. Entretanto, somente quando excluídos quatro participantes que brincaram durante o planejamento foi obtida uma diferença estatisticamente significativa, posto que os participantes que planejaram colaborativamente obtiveram valores $13 \%$ maiores para a variável outcome em comparação a quando planejaram individualmente. Algumas interpretações são apresentadas para tais resultados.

Primeiro, o número de 17 participantes foi uma limitação para o alcance de resultados estatisticamente significativos. Com a escolha pela pesquisa em um contexto escolar intacto e com estudantes que frequentavam a aula de L2 juntos, o número de 30 participantes, que é recomendado para pesquisas que envolvem estatística (LARSON-HALL, 2010) não pode ser alcançado. Segundo, os resultados não estatisticamente significativos para as medidas fluência e acurácia podem ser, em parte, explicados pelo enfoque dado durante o planejamento colaborativo. A análise da transcrição das interações em grupos indicou que os estudantes parecem ter se concentrado em aspectos do gênero textual, isto é, houve uma preocupação com o planejamento macro (LEVELT, 1989) da mensagem (i.e., direcionamento ao interlocutor específico ou à sequência lógica); logo, aspectos de microplanejamento que compõem a acurácia podem ter sido penalizados, em consonância com os efeitos de trade-off (FOSTER; SKEHAN, 1996) observados em estudos anteriores (GUARà-TAVARES, 2009; MEHNERT, 1998; SKEHAN et al., 2012). Nessa perspectiva, a redução no número de palavras em português e o valor mais alto de outcome das mensagens planejadas de forma colaborativa parecem sustentar tal conjectura. Esse enfoque primário da atenção para o significado da mensagem oral é condizente com o arcabouço teórico do ensino e aprendizagem por tarefas (ELLIS, 2005; SKEHAN, 2003), então, uma terceira possibilidade para explicar tais resultados é que o tempo de 12 minutos para o planejamento colaborativo não foisuficiente para gerar diferenças estatisticamente significativas no desempenho dos participantes em cada uma das condições.

Quarto, contrariamente ao conceito teórico que preconiza a proficiência mais elevada na L2 como aspecto positivo nas relações entre aprendizes (STORCH, 2002), tal diferença pode ter sido um entrave para o trabalho colaborativo deste estudo. Isso porque é notório o impacto da proficiência na L2 na produção (ORTEGA, 2005); portanto, esperava-se que, ao planejar de forma colaborativa, aprendizes com diferentes níveis de proficiência na língua se engajassem em diálogos colaborativos (SWAIN, 1985). Apesar de os participantes não terem tido sua proficiência em inglês testada, desde o momento das observações de aulas notou-se uma grande heterogeneidade no grupo em relação ao domínio do inglês, o que, de fato, refletiu nas mensagens orais gravadas. Todavia, tal pluralidade 
parece ter sido um obstáculo em alguns grupos, tendo em vista que a ajuda por alguns aprendizes era constantemente solicitada durante o planejamento em grupo, ao ponto de, em alguns momentos, tais aprendizes (aqueles que faziam o papel de expert na interação) demonstrarem sinais de irritação. Assim, é notório que diferenças muito marcadas em relação a níveis de proficiência na L2 podem impactar negativamente os resultados do trabalho colaborativo (LYNCH; MACLEAN, 2000).

Em síntese, a análise dos dados quantitativos indica uma tendência a favor do trabalho colaborativo para as três medidas de produção oral analisadas, sendo que essa diferença entre as variáveis de planejamento mostrou-se estatisticamente significativa para a medida outcome quando os dados de quatro participantes que não seguiram as instruções da tarefa foram excluídos do teste. Em face desses resultados e análises, a hipótese relacionada à pergunta de pesquisa 1 confirma-se parcialmente, em razão de que o planejamento colaborativo resultou, ainda que de forma não estatisticamente significativa para todas as variáveis, em performances mais fluentes, gramaticalmente precisas e apropriadas do que o desempenho oral dos mesmos alunos após planejarem individualmente.

\subsection{Dados Qualitativos}

Como a coleta de dados envolveu dois momentos distintos, sendo que os participantes responderam ao questionário de percepção sobre a tarefa ao final de cada um deles, os resultados referentes à percepção do planejamento serão apresentados em dois blocos.

Em relação à percepção a respeito da forma de planejamento, foi questionada ao voluntário sua preferência pelomodelo de planejamento oposto ao realizado. Por exemplo, quem planejou em grupo respondeu à seguinte pergunta: "você preferiria ter planejado sozinho(a)? Por quê? Por que não? (seus colegas de sala NÃO terão acesso a essa informação)".

O primeiro momento da coleta mobilizou oito participantes, que cumpriram a ação individualmente, enquanto outros noveplanejaram em trios. Dos oito, cincoindicaram a preferência pelo planejamento com colega(s); grande parte das justificativas apontou que o colega poderia: "saber alguma palavra que eu tenho dúvida" (P. 8) ou "me ajudar a formular melhor a frase" (P. 6). Esse enfoque no aspecto semântico e sintático está em sintonia com a hipótese da produção (SWAIN, 2001; SWAIN; LAPKIN, 2005), dado que, ao se deparar com o desafio da produção na L2 e notar suas lacunas linguísticas, o aprendiz recorreria a recursos externos para solucionar aspectos de forma. Um dos estudantes que assinalou a preferência pelo planejamento individual salientou que gostou de montar a sua própria desculpa; portanto, o planejamento colaborativo não seria positivo. É interessante observar que esse posicionamento foca o significado da mensagem, e não a forma, o que pode indicar que tal participante tenha proficiência mais alta em inglês e não perceba, assim, possíveis aspectos positivos da interação (LYNCH; MACLEAN, 2000). 
Do grupo que planejou com os colegas, sete, dos nove participantes, apontaram a preferência pelo planejamento colaborativo. Ao justificar tal escolha, ressaltaram que os colegas: "ajudaram com palavras" (P.16), ou que "com a ajuda do grupo eu consegui elaborar algo melhor" (P.12); além disso, P. 13 destacou: "eu gosto de ajudar os outros". Novamente, "pensar só na minha (desculpa)" (P.15) foi a razão apontada pelos dois aprendizes que prefeririam ter planejado individualmente. A ajuda que os colegas prestaram, principalmente do ponto de vista lexical com traduções, foi percebida na transcrição das interações dos grupos e corrobora o benefício de poder dialogar com os colegas a fim de preencher lacunas linguísticas percebidas (SWAIN, 2000). Dessa forma, no total, 12 (70,6\%) dos 17 participantes afirmaram a preferência pelo planejamento colaborativo.

Após realizarem a segunda tarefa, quando os participantes planejaram sob a condição inversa ao primeiro momento, foi perguntado se eles gostaram e o quanto gostaram de realizar a segunda tarefa. Dos aprendizes que planejaram em grupo, sete de oito estavam muito felizes, enquanto somente quatro de nove estudantes que planejaram individualmente assinalaram essa opção. Ao justificar sua posição neutra em relação à tarefa com planejamento individual no segundo momento, P. 17 ressaltou: "sim, pois deu para perceber um pouco minhas dificuldades. Mas não havia meus colegas para me ajudar com elas". Essa posição começa com a afirmação de que P.17 gostou de fazer a tarefa, pois o planejamento individual possibilitou perceber suas lacunas linguísticas ${ }^{15}$ (SWAIN, 2000); porém, a ausência da cooperação para a realização de tarefas (FOSTER; OHTA, 2005), no caso, o planejamento colaborativo, mostrou-se como um entrave.

Portanto, com base nos dados qualitativos apresentados, à segunda pergunta da pesquisa - "Qual a percepção dos alunos participantes sobre as diferentes condições de performance?” - responde-se que, embora as duas condições de planejamento estratégico sejam percebidas de forma positiva, os participantes mostraram preferência pelo planejamento colaborativo.

Finalmente, também foi perguntado no questionário de percepção o grau de satisfação com o uso do WhatsApp pelo celular para realizar a tarefa e 15, dos 17 participantes, indicaram a opção "feliz" ou "muito feliz". Em relação a isso, as justificativas centraram-se no fato de o uso ter sido criativo e diferente do que é feito normalmente na escola. Nas palavras do P.16, "eu adoro mexer no celular então foi cool". Tal entusiasmo no uso do celular para a tarefa reflete a presença desse aparelho na vida do adolescente contemporâneo. O questionário de perfil indicou que muitos aprendizes jogam games (possivelmente no celular) e usam aplicativos para aprender idiomas e, por fim, durante as observações de aula o primeiro autor notou o constante uso do celular pelos participantes. $\mathrm{O}$ fato de estudantes sentirem-se mais à vontade em compartilhar suas produções através do WhatsApp, ponto levantado por Weissheimeret al. (2018), parece ser sustentado pelos dados qualitativos do estudo empreendido. 


\section{Considerações finais}

As tarefas, como abordagem de aprendizagem em L2, ocupam um papel proeminente na área de aquisição de L2, e é em razão dessa relevância que estudos como o recém-apresentado procuram investigar sua aplicabilidade no contexto escolar no Brasil, em especial o da escola pública. Ciente dessa demanda, o objetivo desta investigação foi o de analisar o impacto do planejamento individual e colaborativo na performance de uma tarefa oral em L2 por 17 adolescentes aprendizes de inglês. De modo geral, o planejamento estratégico colaborativo em grupos apresentou melhores resultados para outcome em relação ao planejamento individual, resultado alinhado à literatura (SWAIN, 1985, 2001; SWAIN; LAPKIN, 2005) e estudos empíricos (BENISS; BAZZAZ, 2014; XHAFAJet al., 2011) analisados.

Os dados quantitativos mostraram uma consistente tendência de o planejamento estratégico colaborativo produzir mensagens orais mais fluentes, gramaticalmente precisas e apropriadas, confirmando queos desafios envolvidos na produção oral de uma L2 (KORMOS, 2014) podem ser de certa forma atenuados quando pelo menos parte dos processos de conceitualização e formulação (LEVELT, 1989) acontecem anteriormente ao momento da articulação da fala. Além disso, os valores de desviopadrão mostraram variações menores nas medidas orais analisadas para as mensagens produzidas após o planejamento colaborativo, indicando que, ao planejar colaborativamente, a produção do grupo tende a ser mais uniforme, o que é um fato pedagogicamente interessante. Logo, esses resultados corroboraram parcialmente a hipótese do impacto do planejamento colaborativo.

Como apresentado, os dados qualitativos indicaram que os participantes perceberam a tarefa oral como um instrumento para o desenvolvimento da habilidade comunicativa em L2 de forma criativa. Houve uma percepção consistentemente positiva em relação ao uso dos celulares e do WhatsApp para a tarefa,pois o celular está intrinsecamente ligado ao cotidiano da população jovem. Finalmente, ainda, a maioria dos participantes indicou a preferência pelo planejamento colaborativo, tendo como justificativa a ajuda proporcionada pelos colegas para suprir demandas linguísticas na L2, que, como visto, foram além do campo semântico.

No tocante às implicações pedagógicas desta pesquisa, a implantação pontual de uma tarefa com o uso de celulares mostrou grande aceitação pelos participantes, o que sustentaa afirmação de que tal movimento pode ser replicado em sala de aula, com a inserção de tarefas em L2 de forma gradual. Além disso, embora por demandas da pesquisa não foi permitido que aprendizes repetissem a tarefa, o uso de tarefas similares com ferramentas assíncronas, ${ }^{16}$ como o WhatsApp, podem aumentar a confiança do aprendiz e resultar em melhor performance (cf. WEISSHEIMER et al., 2018) com o uso da repetição; dessa forma, tarefas similares podem ser adaptadas como lição de casa, por exemplo. No que concerne ao planejamento, o uso da L1 mostrou-se potencialmente 
significativo em possibilitar discussões metalinguísticas contextualizadas sobre a L2. O surgimento dessas discussões, no entanto, nem sempre resultou na solução almejada pelo grupo. Em sala de aula, tais discussões podem ser anotadas pelo professor durante o planejamento e utilizadas no momento de pós-tarefa (SKEHAN, 1996), direcionando a atenção de todos os alunos para aspectos da forma que emergiram da necessidade comunicativa dos próprios alunos.

Também convidamos nossos colegas professores a olhar, cada vez mais, para a produção como parte do processo da aprendizagem de L2 e não apenas como seu produto para avaliação, considerando-se que os resultados e discussões levantados por esta pesquisa ressaltam o papel da produção como fator desencadeador com vistas a alcançar tal aprendizagem.

Por fim, retomamos a observação inicial feita pelo professor do colégio na introdução, de que aprendizes de inglês não estão acostumados a planejar estrategicamente para tarefas na escola. Tal comentário não é aqui percebido como restritivo, mas sim como uma janela de oportunidade para que professores possam implementar tarefas em L2, sejam elas orais ou escritas, mediadas por planejamento colaborativo e individual na escola. Apesar de possuírem características próprias, essas duas formas de planejar são aqui entendidas como duas faces da mesma moeda e, pelo viés pedagógico, ambas ajudam o aprendiz a perceber lacunas linguísticas na L2, ir além na sua produção, e refletir sobre a língua.

\section{Agradecimentos}

Aos revisores anônimos e editores que apresentaram valiosas sugestões e comentários. Aos colegas que colaboraram na coleta de dados, bem como aos participantes voluntários da pesquisa.

Este trabalho foi realizado com apoio da Coordenação de Aperfeiçoamento de Pessoal de Nível Superior - Brasil (CAPES), Código de Financiamento 001.

Notas

1. O termo L2 é usado em referência ao inglês ensinado no Brasil. Há uma vibrante discussão acerca dos termos: língua estrangeira, segunda língua, língua adicional, entre outras, que ocorre nesse momento no país. No entanto, com base na literatura que dá suporte a esta pesquisa e ao amplo o uso do termo L2 na área de secondlanguageacquisition optou-se pelo termo segunda língua (L2).

2. Metacognição parece ser crucial para que o uso de estratégias seja de fato útil. Oxford (2013) argumenta que talvez uma das tarefas mais essenciais de professores de L2 seja justamente desenvolver, em seus alunos, as capacidades de entender e controlar processos cognitivos.

3. De acordo com Schmidt (2001, p. 29), o conceito de noticingé "o registro consciente da ocorrência de algum evento" que está aquém do nível do entendimento. Essa percepção consciente no nível superficial - noticing - é vista por Schmidt como facilitadora no desenvolvimento de uma L2.

4. Acurácia é entendida como a medida que analisa a fala que não apresenta desvios da forma padrão em relação ao uso da fala pelo nativo/a da língua, não em relação à gramática prescritiva. A medida de fluência,conforme com Tavakoli e Skehan (2005), está relacionada à: a) velocidade, b) pausas e c) reparos na fala. Complexidade é uma medida cuja escolha de parâmetro - gramatical ou lexical - tem variado de acordo 
com diferentes pesquisadores (MICHEL, 2017). Em geral, essa medida aponta o grau de complexidade da fala baseada no parâmetro escolhido.

5. Tradução do original: pushed output.

6. O termo "grupo" refere-se ao agrupamento com mais de dois aprendizes, em oposição ao trabalho em pares.

7. Esse trabalho foi aprovado pelo comitê de ética da Universidade Federal de Santa Catarina,em fevereiro de 2017, sob o seguinte protocolo: 1.985.268.

8. No questionário de perfil, $65 \%$ dos participantes reportaram terem estudado inglês no CA por cinco anos ou mais.

9. Para maiores detalhes a respeito doestudo piloto, cita-se o artigo que reflete esse estudo como processo de adaptação e refinamento advindo da presente pesquisa (ZACCARON; D’ELY; XHAFAJ, 2018).

10. A análise da interação em grupo e as respostas ao questionário do estudo piloto indicaram que o intervalo de 10 minutos, descrito por Mehnert (1998) como adequado para o planejamento e utilizado como padrão em estudos similares, não seria suficiente para planejar as tarefas.

11. Esta pesquisa contou com 10 ajudantes para a coleta de dados, em sua maioria estudantes da graduação ou pós-graduação em Letras Inglês, colegas do primeiro autor. Esses colaboradores foram fundamentais em garantir o sucesso da coleta num escasso intervalo de tempo, as duas aulas seguidas de L2, e também em auxiliar os alunos que preferiram não usar seus próprios celulares ( 1 aluno) ou que tiveram problema com a bateria do aparelho (1 aluna).

12. AS-Unit, de uma forma geral, é uma unidade de fala composta de uma oração independente ou uma oração dependente e suas subordinadas. Para uma análise detalhada sobre esta segmentação, ver Foster, Tonkyn e Wigglesworth (2000).

13. Os avaliadores, professores de inglês como L2 por pelo menos cinco anos, já tinham conduzido esse tipo de trabalho para estudos anteriores.

14. Tendo em vista que o número de participantes no segundo momento reduziu para 13, foi usada a versão não paramétrica, Wilcoxon, do teste $\mathrm{T}$ de amostras pareadas. $\mathrm{O}$ número mínimo aconselhável de participantes para uso da versão paramétrica é 15.

15. A partir do conceito de noticing, cunhado por Schmidt (2001), Swain (2000) propôs que, enquanto aprendizes produzem língua, eles também podem noticethe gap; ou seja, podem perceber lacunas no seu conhecimento da língua estrangeira que precisam ser preenchidas.

16. Ferramentas tecnológicas assíncronas são aquelas que, diferentemente das síncronas (por exemplo, um chat room), não exigem que os interactantes estejam conectados ao mesmo tempo. É possível deixar uma mensagem que será lida (e talvez respondida) pelo interlocutor em um momento futuro.

\section{Referências}

ANDERSON, Neil J. The role of metacognition in second language teaching and learning. ERIC Digest, 2002. Disponível em: https://eric.ed.gov/?id=ED463659. Acessoem: 14 junho 2019.

BALEGHIZADEH, Sasan. The effect of pair work on a word-building task. ELT Journal. v. 64, n. 4, p. 405-413, 2010.

BATSTONE, Rob. Language form, task-based language teaching, and the classroom context. ELT Journal, v. 66, n. 4, p. 459-467, 2012.

BEI, Gavin Xiaoyue. Effects of immediate repetition in L2 speaking tasks: a focused study. English Language Teaching. v. 6, n.1, p. 11-19, 2013. 
BENISS, Aram Reza Sadeghi; BAZZAZ, VahidEdalati. The impact of pushed output on accuracy and fluency of Iranian EFL learners' speaking. Iranian Journal of Language Teaching Research. v. 2, n. 2, p. 51-72, 2014.

BYGATE, Michael. Effects of task repetition on the structure and control of oral language. In: BYGATE, Michael; SKEHAN, Peter; SWAIN, Merrill. (ed.). Researching pedagogic tasks - second language learning and testing. Harlow: Longman. 2001, p. 23-48.

D’ELY, Raquel C. S. F.A focus on learners' metacognitive processes: the impact of strategic planning, repetition, strategic planning plus repetition, and strategic planning for repetition on L2 oral performance. Tese (Doutorado em Inglês: Estudos Linguísticos e Literários) - Programa de Pós-Graduação em Inglês, Universidade Federal de Santa Catarina. Florianópolis, 2006. Disponível em: https://repositorio.ufsc.br/handle/123456789/89407. Acesso em: 22 outubro 2018.

D’ELY, Raquel; MOTA, Mailce; BYGATE, Martin. Strategic planning and repetition as metacognitive processes in task performance: Implications for EFL learners' speech production. In: WEN, Zhisheng (Edward); AHMADIAN, Mohammad Javad. (ed.) Researching L2 task performance and Pedagogy. in honour of Peter Skehan. John Benjamins, 2019, p. 199-228.

DORNYEI, Zoltan. Research Methods in Applied Linguistics: Quantitative, qualitative and mixed methodologies. UK: Oxford University Press, p. 336, 2007.

ELLIS, Rod. Instructed Second Language Acquisition: a Literature Review. Auckland UniServices Limited. Auckland: New Zealand, 2005.

FARIAS, Priscila Fabiane. Task-Test: what lies beyond implementing a task-based assessment? Comparing learners' performance and unveiling learners' perception in a testing situation. Dissertação (Mestrado em Inglês: Estudos Linguísticos e Literários) -Programa de Pós-Graduação em Inglês, Universidade Federal de Santa Catarina. Florianópolis, 2014. Disponível em:https://repositorio.ufsc.br/ handle/123456789/123320. Acesso em: 14 novembro 2018.

FORTKAMP, Mailce Borges Mota. Working memory capacity and L2 speech production: an exploration study. Tese (Doutorado em Inglês: Estudos Linguísticos e Literários) - Programa de Pós-Graduação em Inglês, Universidade Federal de Santa Catarina. Florianópolis, 2000. Disponível em:http://repositorio.ufsc.br/ xmlui/handle/123456789/78287. Acesso em: 10 julho 2019.

FOSTER, Pauline; OHTA, Amy Snider. Negotiation for meaning and peer assistance in second language classrooms. Applied Linguistics. v. 26, n. 3, p. 402-430, 2005.

FOSTER, Pauline; SKEHAN, Peter. The influence of planning and task type on second language performance. Studies in Second Language Acquisition. v.18, p. 299-323, 1996.

FOSTER, Pauline; TONKYN, Alan; WIGGLESWORTH, Gillian. Measuring spoken language: a unit for all reasons. AppliedLinguistics. v. 21 n. 3, p. 354-375, 2000.

GUARÁ-TAVARES, Maria da Glória. The relationship among pre-task planning, working memory capacity, and L2 speech performance: a pilot study. Linguagem \& Ensino. v. 12, n. 1, p. 165-194, 2009.

GUARA-TAVARES, Maria da Glória. Learners' processes during pre-task planning and Working Memory Capacity. Ilha do Desterro. v. 60, n. 1 p. 79-94, 2016.

HAN, Turgay; KESKIN, Firat. Using a mobile application (WhatsApp) to reduce EFL speaking anxiety. Gist Education and Learning Research Journal. n. 12, Jan.-Jun, p. 29-50, 2016. 
HYDE, Martin. Pair work - A Blessing or a Curse?: An Analysis of pair work from pedagogical, cultural, social and psychological perspectives. System, v. 21, n. 3, p. 343-348, 1993.

IZUMI, Shinichi. Comprehension and production processes in second language learning: in search of the psycholinguistic rationale of the output hypothesis. Applied Linguistics. v. 24, n. 2, p. 168-196, 2003.

KORMOS, Judit. Speech Production and Second Language Acquisition. Routledge, 2014.

KOWAL, Maria; SWAIN, Merrill. Using collaborative language production tasks to promote students' language awareness. Language Awareness. v. 3, n. 2, 1994, p. 73-93.

LARSON-HALL, Jenifer. A guide to doing statistics in second language research using SPSS. New York: Routledge, 2010.

LEVELT, Willem Johannes Maria. Speaking: From intention to articulation. Cambridge, MA: The MIT Press, 1989.

LI, Lanlan; CHEN, Jiliang; SUN, Lan. The effects of different lengths of pretask planning time on L2 learners' oral test performance. TESOL Quarterly. v. 49, n.1, p. 38-66, 2015.

LIMA, Marília dos Santos; COSTA, Patrícia da Silva Campelo. O diálogo colaborativo como ação potencial para a aprendizagem de línguas. TrabalhosemLinguísticaAplicada. v. 49, n. 1, p. 167-184, 2016.

LONG, Michael. Focus on form: A design feature in language teaching methodology. In DE BOT, Kees; GINSBERG, Ralph; KRAMSCH, Claire (ed.). Foreign language research in crosscultural perspective. Amsterdam: John Benjamin, 1991, p. 179-192.

LONG, Michael. Focus on form in task-based language teaching. In: LAMBERT, Richard; SHOHAMY, Elana (ed.), Language policy and pedagogy. Amsterdam: Benjamins, 2010, p. 456.

LONG, Michael. Second language acquisition and task-based language Teaching. Oxford: Wiley-Blackwell, 2015.

LOEWEN, Shawn; SATO, Masatoshi. Interaction and instructed second language acquisition: State-of-the-art. Language Teaching. v. 1, n. 3, p. 285-329, 2018.

LYNCH, Tony; MACLEAN, Joan. Exploring the benefits of repetition and recycling of a classroom task. Language Teaching Research. v. 4, n. 3, p. 221-250, 2000.

MCLAUGHLIN, Barry. Theories of Second Language Learning. London: Edward Arnold, 1987.

MEHNERT, Uta. The effects of different lengths of time for planning on second language performance. Studies in Second Language Acquisition. v. 20, p. 83-108, 1998.

MICHEL, Marije. Complexity, Accuracy and Fluency in L2 Production. In: LOEWEN, Shawn; SATO, Masatoshi. The Routledge Handbook of Instructed Second Language Acquisition. New York: Routledge, 2017, p. 50-68.

ORTEGA, Lourdes. Planning and focus on form in L2 oral performance. Studies in Second Language Acquisition. v.21, n. 1, p.109-148, 1999.

ORTEGA, Lourdes. What do learners plan? Learner-driven attention to form during pretask planning. In: ELLIS, Rod (Ed.). Planning and task performance in a second language. Amsterdam and Philadelphia: John Benjamins, 2005, p. 77-109.

ORTEGA, Lourdes. Understanding second language acquisition. London: Hodder Education, 2009. 
OXFORD, Rebecca L. Teaching and researching language learning strategies.New York: Routledge, 2013.

PAIVA, Vera Lucia Menezes de Oliveira. The complexity of online collaborative writing. Horizontes de Linguística Aplicada. v. 14, n.1, p. 15-30, 2015.

PANG, Francine; SKEHAN, Peter. Self-reported planning behaviour and second language performance in narrative retelling. In: SKEHAN, Peter (ed.). Processing Perspectives on Task Performance. Amsterdam: John Benjamins, 2014, p. 95-128.

PALOTTI, Gabriele. CAF: Defining, refining and differentiating constructs. Applied Linguistics. v. 30, n. 4, p. 590-601, 2009.

RICHARDS, Jack; FARRELL, Thomas. Practice teaching: a reflective approach. New York: Cambridge University Press, 2011.

SCHMIDT, Richard. Attention. In: ROBINSON, Peter (ed.). Cognition and second language instruction. Cambridge: Cambridge University Press, 2001, p. 3-32.

SKEHAN, Peter. A framework for the implementation of task-based instruction. Applied Linguistics. v.17, n. 1, p. 38-62, 1996.

SKEHAN, Peter. A cognitive approach to language learning. Oxford: Oxford University Press, 1998.

SKEHAN, Peter. Task-based Instruction. Cambridge: Cambridge University Press, 2003.

SKEHAN, Peter. The context for researching a processing perspective on task performance. In: SKEHAN, Peter (ed.). Processing Perspectives on Task Performance. Amsterdam: John Benjamins, 2014, p. 1-26.

SKEHAN, Peter; XIAOYUE, Bei; QIAN, Li; WANG, Zhan. The task is not enough: Processing approaches to task-based performance. Language Teaching Research. v. 16, n. 2, p. 170-187, 2012.

SPECHT, Andre. Is strategic planning enough?:investigating the impact of two types of strategy instruction on students' oral planned performance. Tese (Doutorado em Inglês: Estudos Linguísticos e Literários) - Programa de Pós-Graduação em Inglês, Universidade Federal de Santa Catarina. Florianópolis, 2017. Disponível em:https://repositorio.ufsc.br/handle/123456789/187086. Acesso em: 12 outubro 2018.

SPECHT, Andre; D'ELY, Raquel Carolina Souza Ferraz.Planning oral narrative tasks: optimizing strategic planning condition through strategy instruction. ActaScientiarum (UEM), v. 39, n. 2, p. 203- 212, 2017.

STORCH, Neomy. Relationships formed in dyadic interaction and opportunity for learning. International Journal of Educational Research. v.37, n. 3, p. 305-322, 2002.

SWAIN, Merrill. Communicative competence: Some roles of comprehensible input and comprehensible output in its development. In: GASS, Susan; MADDEN, Carolyn (ed.) Input in second language acquisition. Rowley, MA: Newbury House, 1985, p. 235-253.

SWAIN, Merrill. The output hypothesis and beyond: Mediating acquisition through collaborative dialogue. In: LANTOLF, James (ed.) Sociocultural Theory and Second Language Learning. Oxford: Oxford University Press, 2000, p. 97-114.

SWAIN, Merrill. Integrating language and content teaching through collaborative tasks. The Canadian Modern Language Review. v. 58, n.1, p. 44-63, 2001.

SWAIN, Merrill; LAPKIN, Sharon. The evolving sociopolitical context of immersion education in Canada: some implications for program development. International Journal of Applied Linguistics. v. 15, n. 2, p. 169-186, 2005. 
TAVAKOLI, Parvaneh; FOSTER, Pauline. Task Design and Second Language Performance: The Effect of Narrative Type on Learner Output. Language Learning, v. 58, n.2, p. 439-473, 2008.

TAVAKOLI, Parvaneh; SKEHAN, Peter. Strategic planning, task structure and performance testing. In: ELLIS, Rod. (ed.). Planning and task performance in a second language. Amsterdam: John Benjamins, 2005.

VIDAL, Rejane Teixeira. Instrução-focada-na-forma, lingualização e aprendizagem de ILE por aprendizes brasileiros. Revista Brasileira de Linguística Aplicada. v. 10, n.1, p. 179-205, 2010.

WEISSHEIMER, Janaina; CALDAS, Vaneska; MARQUES, Fábio. Using WhatsApp to develop L2 oral production. RevistaLeitura. v. 1, n. 60, p. 21-38, 2018.

XHAFAJ, Donesca C. P.One is good, two is better: Investigating the impact of peerplanning in the oral performance of intermediate L2 English learners. Trabalho de Conclusão de Curso - Letras Inglês. Universidade Federal de Santa Catarina. Florianópolis, 2013.

XHAFAJ, Donesca C. P.; MUCK, Katia E. D’ELY, Raquel C.S.F. The impact of individual and peer planning on the oral performance of advanced learners of English as a foreign language. LinguageméEnsino (UCPel. Impresso). v. 14, n. 1, p. 39-65, 2011.

ZACCARON, Rafael.The more the merrier(?): the impact of individual and collaborative strategic planning on performance of an oral task by young learners of English as an L2 in Brazil.Dissertação (Mestrado em Inglês: Estudos Linguísticos e Literários) -Programa de Pós-Graduação em Inglês, Universidade Federal de Santa Catarina. Florianópolis, 2018a. Disponível em: https://repositorio.ufsc.br/ handle/123456789/189173. Acesso em: 24 julho 2019.

ZACCARON, Rafael. Again and Again: an immediate repetition oral task viewed in light of Swain’s output hypothesis.Dominios de Lingu@gem, v. 12, n. 3, p. 14011427, 2018b.

ZACCARON, Rafael;D’ELY, Raquel C. de S. F.; XHAFAJ, Donesca C. P. Estudo piloto: um processo importante de adaptação e refinamento para uma pesquisa quase experimental em aquisição de 12. Revista do GELNE, v. 20, n. 1, p. 30-41, 2018.

Recebido em: 02/12/2018

Aceito em: 06/06/2019 